\title{
Los Pronombres De Tratamiento En El Paisaje Lingüístico Quindiano (Colombia)
}

\section{Pronouns Of Address In The Linguistic Landscape Of Quindio (Colombia)}

\section{(D) Daniel Guarín ${ }^{1}$}

\author{
Recepción: Enero 19 de 2021 \\ Aprobación: Abril 12 de 2021 \\ Publicación: Junio 30 de 2021
}

Cómo citar este artículo: Guarín, Daniel (2021). "Los Pronombres De Tratamiento En El Paisaje Lingüístico Quindiano (Colombia)".

Miradas, Vol. 16, No 1. pp. 77 - 96 https://doi.org/10.22517/25393812.24862

\section{Resumen}

Esta investigación describe la manera en la cual los pronombres de tratamiento se presentan en el paisaje lingüístico del departamento del Quindío (Colombia). En la investigación se analizaron 99 carteles y anuncios de la ciudad de Armenia y 90 publicaciones de las redes sociales de 3 entidades regionales: la gobernación, la alcaldía y un periódico; desde septiembre hasta diciembre de 2020. Los datos obtenidos indican que los pronombres usted y tú se emplean de manera arbitraria y que el pronombre vos no hace parte del paisaje lingüístico de esta región.

Como conclusión se presenta la direccionalidad de la interacción como una nueva variable que modifica el uso de estos pronombres (tú y usted) en el habla y finalmente se presentan las limitaciones del estudio.

1 Magister en Lingüística. Universidad de Temple University. guarin@temple.edu 
Palabras clave: Paisaje lingüístico, pronombres de tratamiento, Armenia, Quindío, Eje cafetero, español colombiano, variación dialectal

\section{Abstract}

This research describes the way in which pronouns of address are presented in the linguistic landscape of the department of Quindío (Colombia). The investigation analyzed 99 posters and advertisements from the city of Armenia and 90 publications from the social networks of 3 regional entities: the government, the mayor's office, and a newspaper; from September to December 2020.

The data obtained indicate that the pronouns usted and tú are used arbitrarily and that the pronoun vos is not part of the linguistic landscape of this region. In conclusion, the directionality of the interaction is presented as a new variable that modifies the use of these pronouns (tú and usted) in speech and finally the limitations of the study are presented.

Keywords: Linguistic Landscape, Pronouns of address, Armenia, Quindio, Coffee Region, Colombian Spanish, Linguistic Variation

\section{Introducción}

Pensar en paisaje lingüístico, conocido en inglés como Linguistic Landscape, es pensar en la manera en la cual la lengua construye el espacio(BenRafael, 2009; Escobar \& Potowski, 2015; Mooney \& Evans, 2015; Sebba, 2011). Aunque los primeros estudios del paisaje lingüístico datan de hace más o menos 40 años, es tan solo hace unos 10 años que este tema ha alcanzado su máximo desarrollo (Comajoan Colomé \& Long, 2012; Extra \& Barni, 2008; Gorter, 2006; Gorter \& Cenoz, 2017;
Leimgruber et al., 2020). El estudio del signo y sus significados ha sido un tema de interés en diferentes áreas como la geografía urbana, la arquitectura, las ciencias ambientales, la sociología, la antropología, la semiótica y los estudios de comunicación social, entre otros; no obstante, este estudio de signos en la comunidad ha sido adoptado por la sociolingüística bajo el término Paisaje Lingüístico -PL- (Kasanga, 2015). Spolsky (2009) menciona que "El estudio de la señalización pública multilingüe se está convirtiendo en un subcampo de la sociolingüística y de la política lingüística ${ }^{2 "}$ (p. 25). De igual manera, Coulmas (2009) señala que el paisaje lingüístico es un campo fértil para la investigación sociolingüística, pues, en un sentido particular puede indicar la correlación y la covariación del uso de la lengua y la clase social, $\mathrm{y}$, en un sentido más amplio, puede dar cuenta de los vínculos entre la lengua y otros atributos sociales como la religión, la etnicidad, la nacionalidad y la raza.

Esta segunda idea la plantea también Maldonado Sandoval (2020), quien menciona que "el PL no es sólo un índice de etnovitalidad lingüística, sino que también puede dilucidar aspectos económicos, sociales, políticos y culturales a nivel local y global" (p. 4). Es por esto que el PL es un tema de interés si se quieren conocer las variaciones que una lengua presenta dentro de un territorio, ya sea bilingüe o monolingüe, pues, de acuerdo con Castillo Lluch \& Sáez Rivera (2013), el estudio del paisaje lingüístico presenta

2 Traducción propia. "The study of public multilingual signage is developing into sub-field of sociolinguistics and of language policy" 
dos líneas de exploración. Por un lado, la investigación del PL se puede llevar a cabo en territorios bilingües donde se presentan dos o más lenguas, como el español y el vasco en Bilbao, España (Fernández Juncal, 2020); el español y el inglés en Florida, EE. UU. (Franco Rodríguez, 2007). Por el otro, se puede hacer un estudio del PL en territorios monolingües con el fin de explorar la realidad lingüística y la manera en la cual la lengua se ve moldeada por fenómenos sociales y culturales, como el estudio sobre nombres de establecimientos comerciales (Hipperdinger, 2020), o del impacto de presencias migratorias en localidad específicas de una ciudad (Bengochea, 2019). Incluso, trabajos recientes conciben el PL como una herramienta pedagógica que se puede aplicar a la enseñanza de lenguas extranjeras. Por ejemplo, el PL de Bogotá, Colombia como recurso para la enseñanza del español (Maldonado Sandoval, 2020); o la presencia del chino en Valencia, España y su aplicación a la enseñanza de español a sinohablantes (Ma, 2019).

El paisaje lingüístico es relevante para el estudio de una comunidad de habla, pues, como lo señalan Mooney \& Evans (2015), aunque muchos signos con los que nos topamos a diario parecen normales e inconsecuentes, estos construyen el espacio en el que vivimos, comunican mensajes $\mathrm{y}$ transmiten información ideológica, "la lengua y otras características semióticas nos ayudan a entender el tipo de espacio en el que estamos" (Mooney \& Evans, 2015, p. 87). De igual manera, Backhaus (2007) comenta que el PL no sólo indica en qué lugar del mundo uno se encuentra y qué lengua se habla en el territorio, sino que también "provee una perspectiva única de la coexistencia y competición de diferentes lenguas, su escritura, y la manera en la que interactuaran e interfieren entre sí en un determinado lugar" (p. 145)

El Paisaje Lingüístico, además, se concibe como un fenómeno informativo o simbólico (Rubdy, 2015). El PL informacional se da cuando provee detalles acerca de la composición sociolingüística de las comunidades de habla de un área determinada y de la lengua o lenguas utilizadas para comunicarse, indicando así su diversidad lingüística o falta de ella. A su vez, el PL es simbólico cuando refleja el poder, el prestigio y el estatus de la lengua, o sea, si la lengua es dominante o subordinada, o simboliza la fuerza o debilidad de diferentes grupos.

Por otro lado, se debe fijar una distinción entre los signos oficiales y no oficiales que se encuentran dentro del PL. De acuerdo con Mooney \& Evans (2015), los signos o señales oficiales son aquellas producidas por el gobierno, las entidades locales o los dueños de los establecimientos en los que los signos se presentan. Estos mensajes se pueden enmarcar dentro de lo que los autores denominan un discurso "top down". A su vez, los signos producidos por personas naturales o pequeños grupos o comunidades se suelen asociar con un discurso "bottom up".

Entonces, ¿a qué se debe prestar atención cuando se quiere estudiar el 3 Traducción propia. It provides a unique perspective on the coexistence and competition of different languages and their scripts, and how they interact and interfere with each other in a given place. 
paisaje lingüístico de una comunidad? Si bien no hay una respuesta concreta para esta pregunta, algunos autores concuerdan con que, dependiendo de las motivaciones del investigador, existen diferentes tipos de textos o elementos semióticos que se pueden considerar. El paisaje lingüístico, de acuerdo con la definición dada por Coulmas (2009) es un escenario cultural formado por diferentes agentes. El autor menciona que, si se quiere hacer un análisis del PL, se debe prestar atención a la manera en la cual la información se presenta ante un público teniendo en cuenta el lenguaje utilizado y la significación simbólica, pues esto podría representar las motivaciones e intenciones relativas al contenido de la información.

El paisaje lingüístico encierra todo objeto lingüístico que se enmarca dentro de un espacio público y que puede referir a cualquier signo escrito que se encuentre fuera de áreas privadas (Ben-Rafael, 2009). El PL “capturan la presencia de fragmentos de lenguaje escrito visibles públicamente: vallas publicitarias, señales de tráfico y de seguridad, letreros de tiendas, grafitis y todo tipo de otras inscripciones en el espacio público, tanto de producción profesional como artesanal" (Blommaert, 2013, p. 1). Es decir, en el paisaje lingüístico de una comunidad se enmarca cualquier elemento escrito que esté abierto al público, desde señales de tránsito hasta nombres de calles, tiendas o colegios:

El estudio de PL se centra en analizar estos ítems según el lenguaje utilizado, su relevancia relativa, aspectos sintácticos o semánticos. Desde la premisa de la sociología del lenguaje, los hechos del lenguaje que marcan el espacio público deben verse como hechos sociales cuyas variaciones deberían relacionarse con fenómenos sociales más generales ${ }^{4}$. (BenRafael, 2009, p. 40)

Además, algunos autores manifiestan que el estudio del paisaje lingüístico no se debe centrar sólo en el lenguaje escrito. Gorter \& Cenoz (2017), por ejemplo, mencionan que en el análisis del PL se deben incluir elementos multimodales, semióticos, visuales, e incluso orales. Igualmente, en los últimos años, con el desarrollo tecnológico, el estudio del Paisaje Lingüístico se ha extendido a espacios virtuales. Ivkovic \& Lotherington (2009), por ejemplo, presentan una conceptualización del PL en el mundo virtual y presentan la evolución que las lenguas han tenido al estar en contacto desde el inicio de las plataformas en internet hasta las plataformas multimodales (web 2.0), una revolución comunicativa que, de acuerdo con los autores, ha impactado la diversidad lingüística.

Así, "examinar signos, tanto en el paisaje lingüístico del 'mundo real' como en el paisaje virtual de la red informática mundial, nos muestra cómo las personas crean significados y entienden las contribuciones de otros ${ }^{5}$ " (Mooney \& Evans, 2015, p. 107). Y, de manera similar al paisaje lingüístico del

4 Traducción propia. The study of LL focuses on analyzing these items according to the languages utilized, their relative saliency, syntactical or semantic aspects. From sociology-of-language premise, language facts that landmark the public space are to be seen as social facts the variations of which should relate to more general social phenomena. 5 Traducción propia. 
mundo real, Kasanga (2015) propone clasificar el PL virtual en espacios privados (blogs personales), y públicos (sitios webs de instituciones).

En síntesis, el paisaje lingüístico busca explorar la manera en la cual la lengua o lenguas de una comunidad se presenta en espacios públicos, ya sea de manera real o virtual, y busca dar cuenta de cómo la lengua codifica la identidad de las personas que hacen parte de cierta comunidad. De esta manera, "el estudio del paisaje lingüístico se convierte en una nueva dimensión para mapear y medir la diversidad lingüística" (Extra \& Barni, 2008, p. 25). Así pues, este trabajo busca explorar el paisaje lingüístico del departamento del Quindío en Colombia con base en carteles y anuncios publicitarios en espacios públicos dentro del departamento, y de manera virtual a través de los comunicados de las redes sociales de la gobernación del Quindío, la alcaldía de Armenia y La Crónica del Quindío, uno de los periódicos más populares del departamento, haciendo énfasis en el uso de los pronombres de tratamiento tú, usted y vos. A continuación, se presenta una breve conceptualización de los pronombres de tratamiento y su estudio en el habla quindiana.

\section{Pronombres de tratamiento en el Quindío}

Los pronombres de tratamiento en español les permiten a los hablantes elegir de qué manera dirigirse a su interlocutor. Este fenómeno lingüístico presenta una amplia complejidad desde los estudios sociales, pues, a pesar de que existe una concepción básica que plantea el uso de usted en aspectos formales y el uso de $t u ́$ en contextos informales (Brown \& Gilman, 1970), análisis sociolingüísticos e interculturales han demostrado que la alternancia de estos pronombres rompe esta dicotomía.

La complejidad de esta alternancia podría residir en que los usos de estos pronombres varían entre España y América, siendo el uso del pronombre vos una característica principal para su diferenciación (Doyle D'Ambrosio, 2004; Lara Bermejo, 2018; Mas Álvarez, 2014; RiveraMills, 2011). A su vez, el uso de los pronombres de tratamiento varía dentro de los mismos países. En Colombia, por ejemplo, los dialectos de las ciudades presentan alternancia entre el uso de estos pronombres (Lindgård, 2016; Montes Giraldo, 1982), aunque, vale aclarar, la variación pronominal en Colombia no solo existe en la ciudad, sino que hay regiones dialectales distinguidas por un mayor o menor uso de "usted, tú, vos".

En Bogotá, por ejemplo, es común escuchar diferentes pronombres para dirigirse a la segunda persona del singular: tú, usted, vos y sumercé (López López, 2015)"event-place":"Bogotá, Colombia", "publisher":"Red Académica del Español como Lengua Extranjera -EnRedELE", "publisher-place": "Bogotá, Colombia", "title":" Diferencias dialectales en el uso de las formas de tratamiento: tú y usted en páginas de Facebook de dos equipos de fútbol de Bogotá","URL":"'https:// spanishincolombia.caroycuervo. gov.colencuentro-lengua-extranjera/ 
ponencias/L\%C 3\%B 3 pez\%20 $L \% C 3 \%$ B 3 pez\% 20 Giovani. pdf"," "author":[\{"family":"López Ló pez", "given":"Giovani",\}], "issued" :\{“date-parts":[["2015"]]\}\}\}], "sc hema":"https://github.com/citationstyle-language/schema/raw/master/cslcitation.json"' , a su vez, su uso varía según algunas variables sociales como la edad, la estratificación socioeconómica, el sexo y la orientación sexual (Bartens, 2003; Cepeda Ruiz, 2014; ColensoSemple, 2008; Montes Giraldo, 1985; Diane R. Uber, 2011; Diane Ringer Uber, 1985). De otra parte, en Medellín, aunque también existe variación entre el uso de los pronombres usted, tú y vos (Jang, 2012), el uso de vos se presenta como una marca de identidad regional al ser el pronombre usado mayormente entre los ciudadanos en conversaciones formales e informales (Flórez, 1953; Jang, 2014) e incluso en las redes sociales (paisaje lingüístico virtual) de la alcaldía de Medellín (Weyers, 2016).

Con respecto al Eje Cafetero, son pocos los estudios que se han realizado en relación con la variación lingüística de esta zona (Agudelo Montoya et al., 2016; Cisneros Estupiñán et al., 2014; García Rodríguez, 2014; Guarín et al., 2015; Idárraga Valencia, 2017; Ospina Castro, 2018; Serna Pinto, 2018). Sobre el uso de pronombres de tratamiento en el Quindío, Guarín (2021) concluye que el habla quindiana se caracteriza por el uso del pronombre usted. También, el autor menciona que el pronombre vos, a pesar de ser muy poco frecuente en la sociedad, se emplea de manera esporádica, y que en los hablantes jóvenes el pronombre tú presenta un mayor uso que el pronombre usted mientras que el pronombre vos desaparece. Lo que, según el autor, se podría concebir como un cambio en tiempo aparente.

Con base en lo anterior, surge la pregunta de investigación ¿de qué manera se presentan los pronombres de tratamiento en el paisaje lingüístico del departamento del Quindío? A continuación, se presenta la metodología de investigación.

\section{Metodología}

El objetivo de esta investigación es describir la manera en la cual los pronombres de tratamiento se presentan en el paisaje lingüístico del departamento del Quindío (Colombia). Si bien un estudio reciente sobre la variación lingüística de este departamento concluye que su habla se caracteriza por el uso del pronombre usted (Guarín, 2020), se podría pensar entonces en la siguiente hipótesis:

I. Si bien estudios cuantitativos sobre el uso de pronombres de tratamiento en el Quindío concluyen que el pronombre característico de la región es usted, el PL del territorio debe reflejar esta variación lingüística, por lo tanto, los carteles y anuncios públicos emplean el pronombre usted.

Así, como ruta metodológica para esta investigación se sigue lo planteado por Kasanga (2015) quien presenta el término "elección de código" (Code choice) y que busca enfocarse en la variedad de una lengua en el Paisaje Lingüístico. De esta manera, se empieza a hacer una observación de los diferentes carteles $\mathrm{y}$ anuncios publicitarios en los espacios 
públicos del departamento $\mathrm{y}$, de manera virtual, en las publicaciones de las redes sociales de la gobernación del Quindío, la alcaldía de Armenia y el periódico regional La crónica del Quindío, en los cuales se haga uso de los pronombres de tratamiento. La observación se llevó a cabo entre septiembre y diciembre de 2020.

Sobre las variables que se pueden contemplar para un estudio sobre el PL, Amos \& Soukup (2020) mencionan dos niveles: nivel físico y nivel de discurso. El nivel físico se refiere a aspectos de la apariencia y presentación material de un signo, es decir, qué anuncio es y dónde se encuentra ubicado; mientras que el nivel del discurso explica las propiedades del texto en sí, es decir, de que se trata el anuncio.

De esta manera, para el paisaje lingüístico real en esta investigación se tendrán en cuenta dos niveles: físico y discursivo. El nivel físico se subdividirá en vallas o pancartas de gran dimensión (billboards), y carteles (avisos de locales, tiendas o negocios). A su vez, el nivel discursivo se subdividirá en publicidad (aquellos volantes, anuncios y carteles que busquen llamar la atención de algún cliente o promocionar un producto), información (carteles que brinden información sobre una entidad, establecimiento público o local en general) y campaña departamental (anuncios hechos por la alcaldía o la gobernación con el fin de informar o concienciar a los habitantes del departamento). La siguiente gráfica representa la metodología de la investigación:
Gráfica 1: diseño metodológico de la investigación

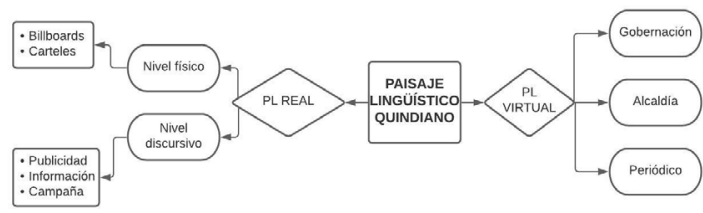

De esta manera, para el análisis del PL real se tendrán en cuenta un total de 100 carteles, vallas publicitarias y anuncios ubicados en diferentes zonas de Armenia. Igualmente, para el análisis del PL virtual se tendrán en cuenta 30 publicaciones por cada subcategoría: gobernación, alcaldía y periódico regional. A continuación, se presentan los resultados obtenidos.

\section{Resultados}

Este apartado presenta los resultados obtenidos en esta investigación cuyo objetivo fue describir la manera en la cual los pronombres de tratamiento se presentan en el paisaje lingüístico del departamento del Quindío. Como se mencionó en el apartado anterior, se hizo un análisis del PL real y el PL virtual. Por un lado, para el análisis del PL real se seleccionaron de manera aleatoria 100 vallas y anuncios distribuidos en la ciudad de Armenia para ser analizados. Por el otro, para el PL virtual se analizaron 30 publicaciones de cada una de las redes sociales de la alcaldía de Armenia, la gobernación del Quindío y del periódico La crónica del Quindío, en los cuales se hace uso de los pronombres de tratamiento, obteniendo un total de 90 publicaciones. 


\section{Paisaje lingüístico}

Para el análisis del PL en el departamento se seleccionaron, de manera aleatoria, 100 carteles y anuncios en los que se hacen uso de los pronombres de tratamiento de manera implícita o explícita. Como se mencionó en el apartado anterior, los niveles considerados para el análisis de datos fueron nivel físico y discursivo. De los 100 carteles seleccionados, un total de 69 utilizan el pronombre $t u$, lo que equivaldría a un $69.7 \%$. Asimismo, 30 carteles emplean el pronombre usted, es decir, el 30.3\%; y no se registró ningún cartel con el pronombre vos. Vale aclarar que uno de los carteles hace uso de ambos pronombres imagen 1 - por lo cual no se incluyó en ninguna categoría $(\mathrm{N}=99)$.

Tabla 1: uso de pronombres en el PL

\begin{tabular}{|l|l|}
\hline Anuncios con tú & $69(69.7 \%)$ \\
\hline $\begin{array}{l}\text { Anuncios con } \\
\text { usted }\end{array}$ & $30(30.3 \%)$ \\
\hline $\begin{array}{l}\text { Anuncios con } \\
\text { VOS }\end{array}$ & $0(0 \%)$ \\
\hline Total & $99(100 \%)$ \\
\hline
\end{tabular}

La imagen 1 muestra un cartel ubicado en el centro de la ciudad de Armenia en el cual se hace uso de los pronombres tú y usted. En la parte superior del cartel se emplea el pronombre usted al informar a los usuarios sobre un beneficio que se ofrece: "reclame". En la parte inferior derecha se leen tres verbos imperativos conjugados con el pronombre tú: retira, consigna y paga.

\section{Imagen 1: uso de ambos pronombres}

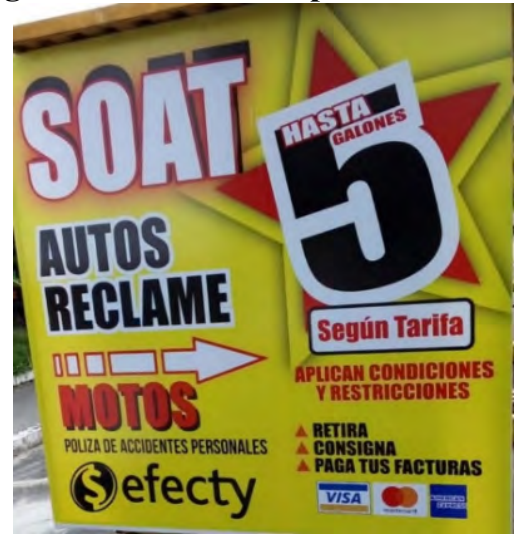

Con respecto a aquellos carteles en los que se hace uso del pronombre usted, la mayoría son publicidad sobre servicios:

- "Cargue aquí su vehículo eléctrico", cartel informativo para usuarios con vehículos eléctricos;

- "¿Quiere comprar? Tarjeta de crédito en 5 minutos", valla publicitaria de entidad bancaria;

- "Visite nuestro apartamento modelo", publicidad de constructora;

- "Remodelar con nosotros es su mejor decisión", cartel en oficina de empresa de remodelación.

Imagen 2: uso de pronombre usted

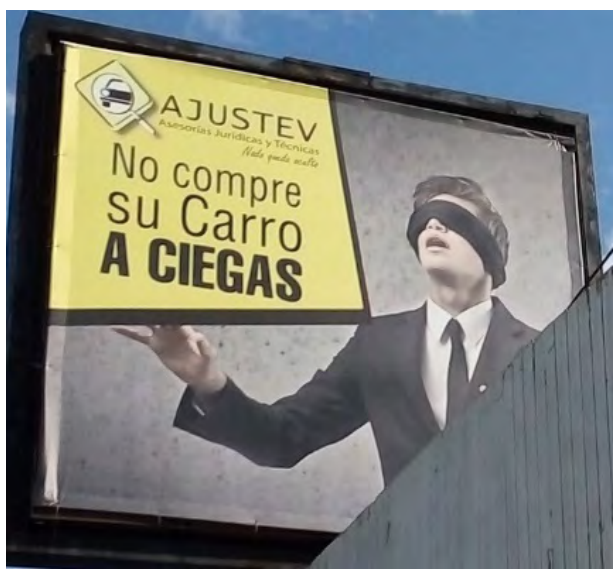


Finalmente, el $\quad 69.7 \%$ de los anuncios encontrados usan el pronombre tú. Algunos ejemplos de carteles y publicidad con el uso de este pronombre son los siguientes:

- "Comparte alegría y brinda en casa", valla publicitaria de bebida alcohólica;

- "Visita nuestro apartamento modelo", "vive el nuevo norte" y "vive en un lugar privilegiado", publicidad de tres constructoras diferentes;

- "Siembra una planta, cosecha vida"; cartel encontrado en un vivero;

- "iVen y descubre el sitio ideal como ninguno!", cartel de un hotel turístico.

Imagen 3: uso de pronombre tú

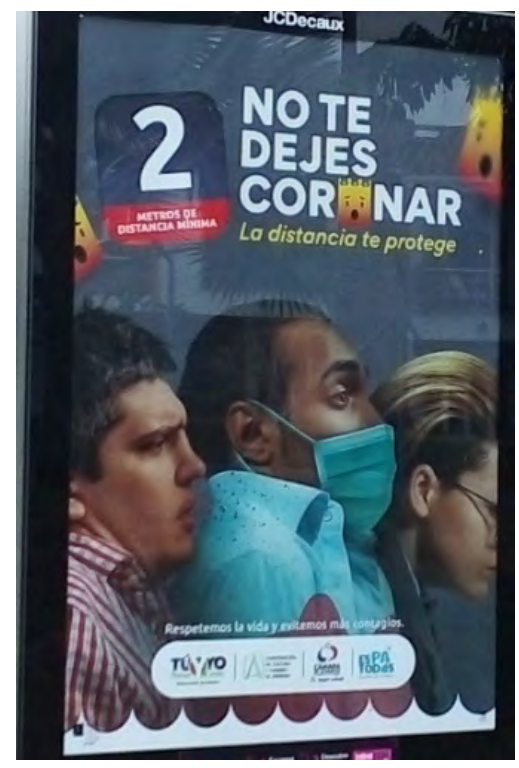

Finalmente, como se mencionó, no se registró ningún caso con el pronombre vos $(0 \%)$. La siguiente gráfica presenta la síntesis de los anuncios encontrados.
Gráfico 2: uso de pronombres en el PL

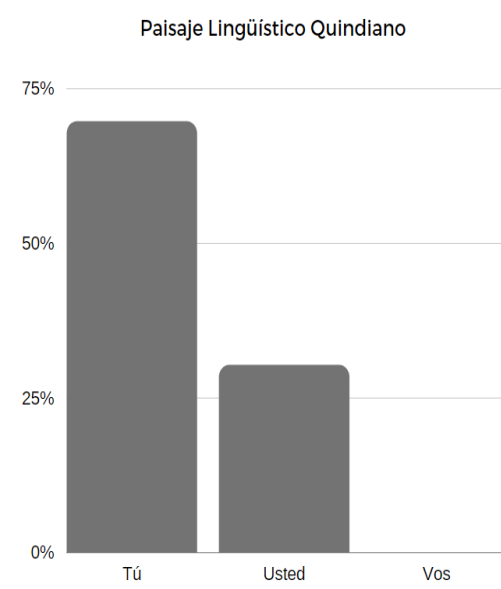

Para el análisis de este PL se tuvieron en cuenta los niveles físico y discursivo. En el nivel fisico se contaron vallas y carteles. En el nivel discursivo se contaron anuncios de publicidad, información y campaña departamental. Esta investigación analizó el nivel físico en las vallas y los carteles distribuidos en el departamento. Por vallas se entienden todos los anuncios de gran tamaño (billboards) que se ubican en una zona aleatoria de la ciudad

Tabla 2: Nivel físico

\begin{tabular}{|l|l|l|l|}
\hline \multicolumn{4}{|c|}{ NIVEL FÍSICO (N=99) } \\
\hline & TÚ (n=69) & $\begin{array}{l}\text { USTED } \\
(\mathrm{n}=30)\end{array}$ & VOS (n=0) \\
\hline VALLAS & $\begin{array}{l}33 \\
(33.3 \%)\end{array}$ & $18(18.1 \%)$ & $0(0 \%)$ \\
\hline CARTELES & $\begin{array}{l}36 \\
(36.3 \%)\end{array}$ & $12(12.1 \%)$ & $0(0 \%)$ \\
\hline
\end{tabular}

Por otro lado, en el nivel discursivo se tuvieron en cuenta todo tipo de vallas o carteles pero se categorizaron en publicidad, como cualquier tipo de cartel cuyo objetivo 
fuera persuadir a los transeúntes de comprar o invertir en algún negocio o producto; información, como aquellos avisos que brindaran información pero no buscaran convencer a los transeúntes; y campañas departamentales, como aquellos anuncios diseñados por parte de entes como la alcaldía, La gobernación o alguna entidad no gubernamental o sin ánimo de lucro.

\section{Imagen 4: ejemplo de campaña departamental}

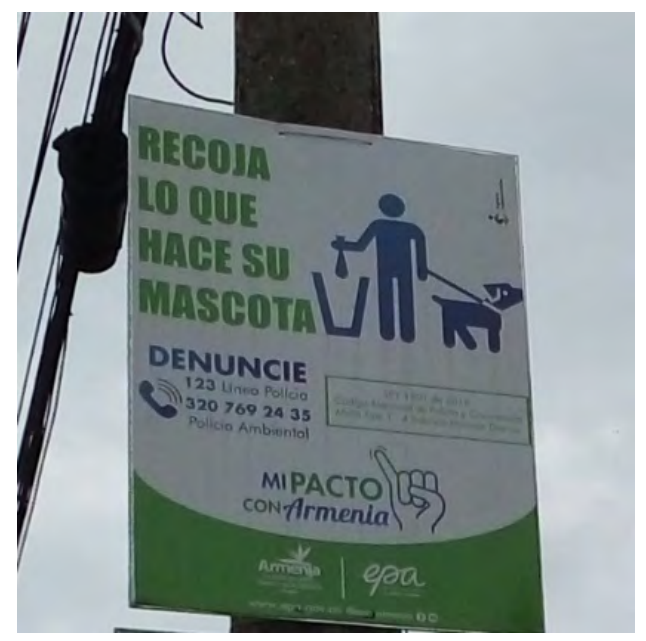

La imagen 4 es un ejemplo del tipo de carteles que se incluyeron en la categoría campaña departamental. Así, carteles como este y los demás anuncios elaborados por la alcaldía, gobernación o demás organizaciones, se incluyeron en esta categoría.
Tabla 3: nivel discursivo

\begin{tabular}{|l|l|l|l|}
\hline \multicolumn{4}{|c|}{ NIVEL DISCURSIVO (N=99) } \\
\hline & $\begin{array}{l}\text { TÚ } \\
(\mathbf{n = 6 5 )}\end{array}$ & $\begin{array}{l}\text { USTED } \\
(\mathbf{n = 3 4 )}\end{array}$ & $\begin{array}{l}\text { VOS } \\
(\mathbf{n = 0})\end{array}$ \\
\hline PUBLICIDAD & $\begin{array}{l}32 \\
(32.3 \%)\end{array}$ & $\begin{array}{l}14 \\
(14.1 \%)\end{array}$ & $0(0 \%)$ \\
\hline INFORMACIÓN & $\begin{array}{l}28 \\
(28.2 \%)\end{array}$ & $\begin{array}{l}12 \\
(12.1 \%)\end{array}$ & $0(0 \%)$ \\
\hline CAMPAÑA & $5(5.5 \%)$ & $8(8.8 \%)$ & $0(0 \%)$ \\
\hline
\end{tabular}

\section{Paisaje lingüístico virtual}

El Paisaje Lingüístico Virtual - PLV — es una extensión que, debido al avance tecnológico, nuevos estudios sociolingüísticos y multidialectales se ha añadido al estudio del PL. El estudio del paisaje lingüístico virtual condensa cualquier tipo de comunicación mediada por internet, ya sea a través de anuncios, páginas web, redes sociales o juegos en línea.

El presente trabajo recogió anuncios de las redes sociales de la gobernación del Quindío, la alcaldía de Armenia y del periódico regional La crónica del Quindío. En cada perfil se identificaron 30 publicaciones en las que se hiciera uso de alguno de los pronombres de tratamiento en torno a los cuales gira esta investigación para su respectivo análisis.

\section{Gobernación del Quindío}

En la página web de la gobernación del Quindío (https:// www.quindio.gov.co/) se encuentra el enlace para acceder a cuenta oficial de Facebook (www.facebook. com/GobernacionQuindio/) de la gobernación. Así, se analizaron ambas páginas para la recolección de datos.

El eslogan de campaña del actual gobierno es "Tú y Yo Somos Quindío", este es un primer 
acercamiento a la manera en la cual los pronombres de tratamiento se emplean en las plataformas de la gobernación. De manera similar, de las 30 publicaciones seleccionadas, 28 emplean el pronombre tú y sólo dos emplean el pronombre usted. Igual que en el PL real, no se registran tokens con el pronombre vos.

Imagen 5: Quédate en casa. Imagen tomada de la página web de la gobernación.

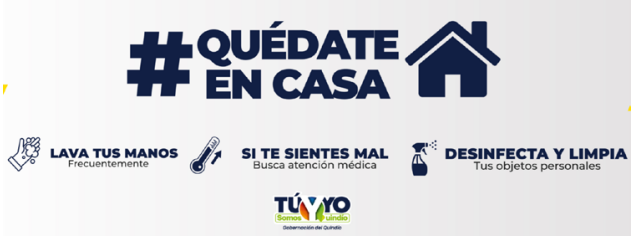

La imagen 5 es una muestra de la manera en la cual la gobernación se dirige a los ciudadanos a través de su página web con información sobre los cuidados respectivos frente al COVID 19. De igual manera, algunos ejemplos de los anuncios de las redes sociales de la gobernación son los siguientes:

- "Vacúnate sin miedo"

- "En temporada de lluvia ¡prepárate ante deslizamientos!"

- "Organización comunal.

Postula tu iniciativa. Descarga el instructivo y formulario de inscripción".

- "¿Qué hacer si filtran tus fotos íntimas?"

- De otra parte, solamente se contaron dos registros del pronombre usted:

- "Para una buena digestión y prevenir enfermedades del corazón, incluya frutas en cada una de las comidas".

- "Para favorecer la salud de los músculos, huesos y dientes, consuma diariamente leche o productos lácteos y huevo".

La siguiente tabla sintetiza los resultados encontrados en la página web y página de Facebook de la gobernación del Quindío.

Tabla 4: tokens en la gobernación del Quindío

\begin{tabular}{|l|l|}
\hline \multicolumn{2}{|c|}{$\begin{array}{c}\text { GOBERNACIÓN DEL } \\
\text { QUINDÍO (N=30) }\end{array}$} \\
\hline TÚ & $28(93.3 \%)$ \\
\hline USTED & $2(6.6 \%)$ \\
\hline VOS & $0(0 \%)$ \\
\hline
\end{tabular}

\section{Alcaldía de Armenia}

De manera similar a la recolección de datos en la gobernación del Quindío, se accedió a la página web de la alcaldía de Armenia (https://www. armenia.gov.co/) y desde allí a las redes sociales oficiales, Facebook (https:// facebook.com/alcaldiaarmenia/) y Twitter (https://twitter.com/ AlcaldiaArmenia).

De las 30 publicaciones seleccionadas, se encontraron un total de 26 publicaciones con el pronombre $t u ́$, cuatro con el pronombre usted, ninguna con el pronombre vos y una publicación en la cual se hace uso de los pronombres usted y tú paralelamente, por lo que se contó en ambas categorías -imagen 6-. 
Imagen 6: uso de dos pronombres en la misma publicación

Alcaldia de Armenia

24 de noviembre a las 12:11.

\#ESPaTodos EI BUS TIC, llegará mañana a la Plaza de Bolivar y al Parque Santander. Si

Lo invitamos a que nos acompañe en esta brigada digital y se suba en este recorrido hacia la transformación digital.

\#SecretariaTIC \#AlcaldiaDeArmenia \#BusTIC

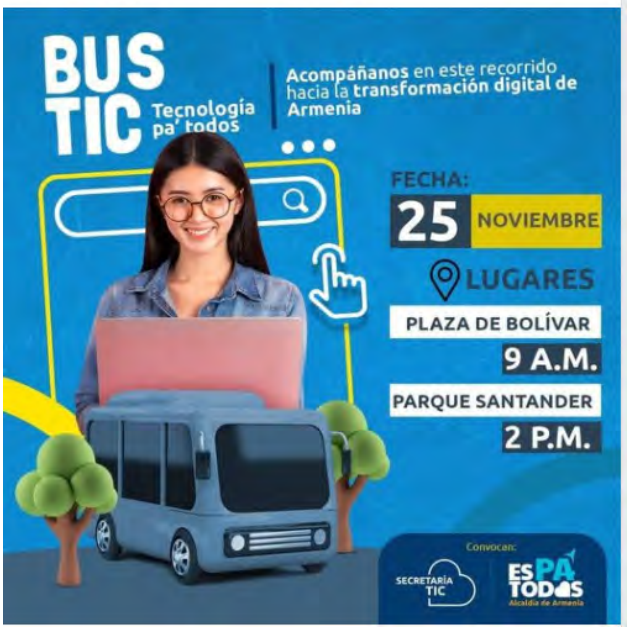

La imagen 6 presenta una alternancia en el uso de los pronombres tú y usted en la misma publicación. El texto utiliza el pronombre usted para dirigirse a los ciudadanos e invitarlos a asistir a la brigada digital del bus TIC: "Lo invitamos a que nos acompañe (...) y se suba en este recorrido". Por otro lado, la imagen emplea el pronombre tú para la misma invitación: "acompáñanos en este recorrido".

Como se mencionó, el $86.6 \%$ de las publicaciones de la alcaldía de Armenia que se contaron en este estudio $(\mathrm{N}=30)$ emplean el pronombre tú. Incluso la imagen del perfil de las redes sociales hace uso de este pronombre: "no te dejes coronar".
Imagen 7: imagen de perfil de las redes sociales de la alcaldía

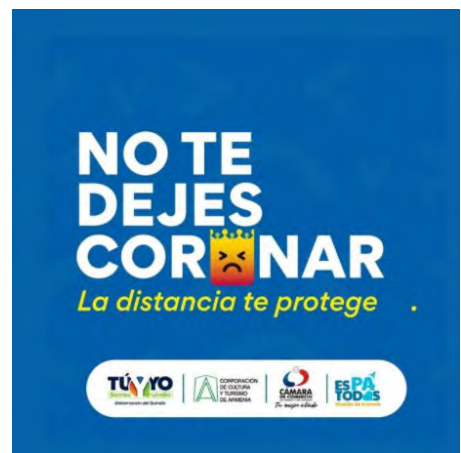

A su vez, tan solo el $13.3 \%$ de las publicaciones emplean el pronombre usted.

Imagen 8: no pague por sus trámites.

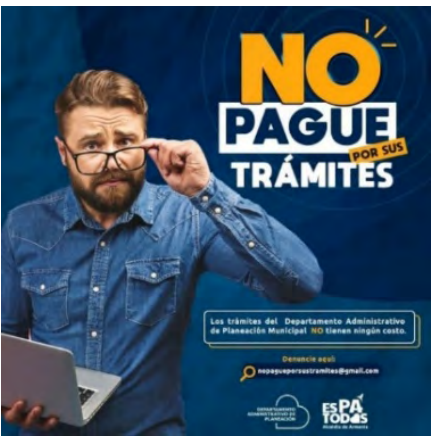

Finalmente, no se evidenciaron publicaciones que hicieran uso del pronombre vos.

Tabla 5: tokens contados en la alcaldía de Armenia

\begin{tabular}{|l|l|}
\hline \multicolumn{2}{|c|}{$\begin{array}{c}\text { ALCALDÍA DE ARMENIA } \\
(\mathbf{N}=\mathbf{3 0})\end{array}$} \\
\hline TÚ & $26(86.6 \%)$ \\
\hline USTED & $4(13.3 \%)$ \\
\hline VOS & $0(0 \%)$ \\
\hline
\end{tabular}

\section{La Crónica del Quindío}

La última entidad que se tomó en cuenta para este estudio sobre el uso de pronombres de tratamiento en 
el paisaje lingüístico virtual quindiano fue La crónica del Quindío, un periódico regional cuya sede principal se encuentra ubicada en Armenia y que presenta noticias e información del departamento del Quindío. Desde la página web del periódico (https:// www.cronicadelquindio.com/) se accedió a las redes sociales oficiales: Facebook (https://www.facebook. com/cronicadelquindio), Twitter (https://twitter.com/croniquindio) e Instagram (https://www.instagram. com/cronicadelquindio/).

En las 30 publicaciones seleccionadas se encontraron 19 publicaciones con el pronombre tú y 11 con el pronombre usted. De manera similar a lo ocurrido en el análisis de las publicaciones de la alcaldía de Armenia, no se evidenciaron usos del pronombre vos y se encontraron dos publicaciones en las cuales se emplean los pronombres tú y usted de manera paralela.

Imagen 9. uso de ambos pronombres en la misma publicación

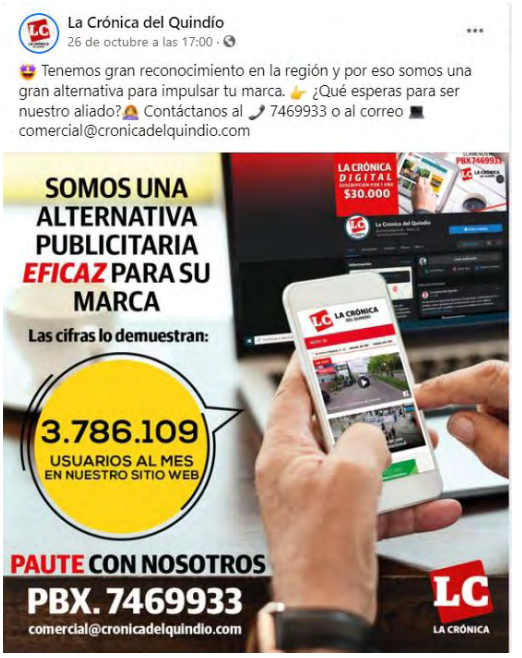

La imagen 9 refleja un caso de curiosidad lingüística pues en la misma publicación se emplean dos pronombres diferentes. Por un lado, en el texto de la publicación se hace uso del pronombre tú: “...somos una alternativa para tu marca. ¿Qué esperas para ser nuestro aliado?" Por otro lado, en la imagen que acompaña la publicación se evidencia el uso del pronombre usted: "somos una alternativa publicitaria eficaz para su negocio (...). Paute con nosotros". Este mismo fenómeno se presenta en otra publicación como se presenta a continuación en la imagen 10 .

Imagen 10: uso de ambos pronombres en la misma publicación.
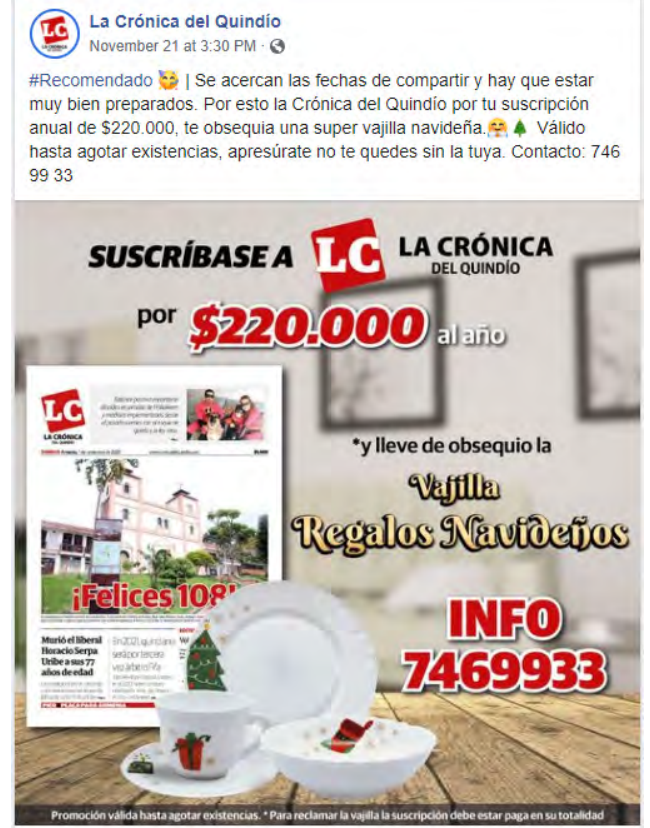

Igual que en el caso anterior, esta publicación emplea el pronombre tú en el texto de la publicación: "la Crónica del Quindío por tu suscripción te obsequia 
una super vajilla. (...) apresúrate no te quedes sin la tuya"; y el pronombre usted en la imagen que la acompaña: "Suscríbase a la Crónica del Quindío (...) y lleve de obsequio la vajilla".

En cuanto a las publicaciones con el pronombre tú $(\mathrm{n}=19)$, en su mayoría se encuentran publicaciones de tipo participativo, como invitaciones a concursos, o publicidad de productos de em-

presas terceras.

Imagen 11: ejemplo de publicación con pronombre tú

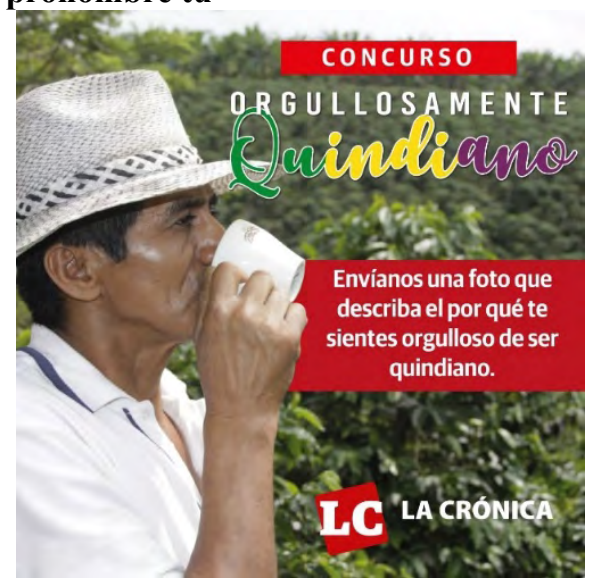

Sobre el uso del pronombre usted $(\mathrm{n}=12)$ se cuentan publicaciones de sondeo o, de manera similar a lo evidenciado con el uso del pronombre tú, de tipo publicitario o participativo.

Imagen 20: ejemplo de publicación con el pronombre usted

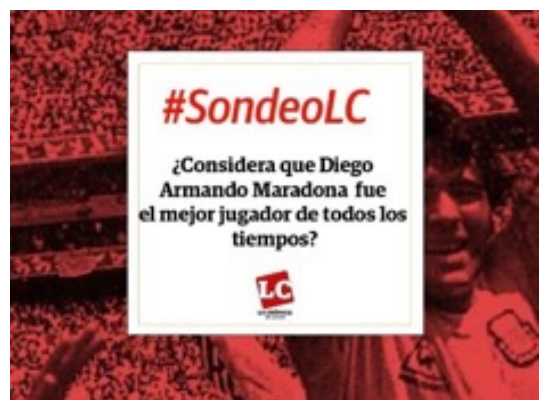

Tabla 6: publicaciones de la Crónica del Quindío

\begin{tabular}{|c|c|}
\hline \multicolumn{2}{|c|}{$\begin{array}{l}\text { LA CRÓNICA DEL QUINDÍO } \\
\qquad(\mathrm{N}=30)\end{array}$} \\
\hline TÚ & $19(63.3 \%)$ \\
\hline USTED & $11(36.6 \%)$ \\
\hline VOS & $0(0 \%)$ \\
\hline
\end{tabular}

La tabla anterior resume los hallazgos en relación con el uso de pronombres de tratamiento por parte del periódico regional La Crónica del Quindío en sus redes sociales. A continuación, en la gráfica 3, se sintetizan los resultados del análisis del paisaje lingüístico virtual quindiano.

Gráfica 3: uso de pronombres de tratamiento en el PLV quindiano

Paisaje Lingüístico Virtual

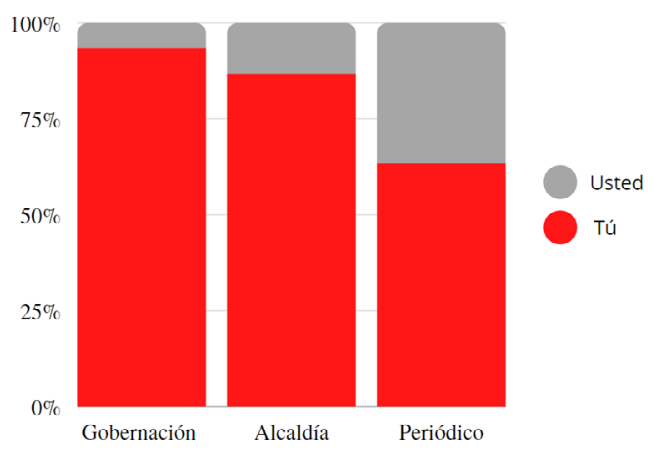

\section{Discusión}

Siguiendo las conclusiones de Guarín (2020) sobre el uso de pronombres de tratamiento, se entiende que "El habla quindiana se caracteriza por el uso del pronombre usted por encima de tú y vos" (p. 34). Así pues, esta investigación buscó corroborar la siguiente hipótesis: 
Si bien estudios cuantitativos sobre el uso de pronombres de tratamiento en el Quindío concluyen que el pronombre característico de la región es usted, el PL del territorio debe reflejar esta variación lingüística, por lo tanto, los carteles y anuncios públicos deben emplear el pronombre usted.

Los resultados obtenidos en esta investigación no corroboran lo propuesto. Como se evidencia en el apartado anterior, en el paisaje lingüístico tan solo el $30.3 \%$ de los anuncios estudiados emplean el pronombre usted. Esta tendencia también se observa en ambos niveles en los que se llevó a cabo la investigación, pues el pronombre es usado un 30.3\% en el nivel físico y un $34.3 \%$ en el nivel discursivo. En su mayoría, los anuncios que se encuentran en la ciudad de Armenia hacen uso del pronombre tú $(69.7 \%$ en el nivel físico y $65.6 \%$ en el nivel discursivo). A su vez, el pronombre vos no presenta ninguna presencia en el PL de la ciudad. Esto último reafirma lo propuesto por Guarín (2020): "El pronombre vos, a pesar de ser muy poco frecuente en la sociedad, se emplea de manera esporádica. No obstante, debido a su poco uso, no se podría reconocer como una característica representativa de la variación dialectal del departamento" (p. 34).

Con respecto a las variables que se tuvieron en cuenta para el análisis, como se presenta en la gráfica 4 , en el nivel discursivo el pronombre usted es mayormente utilizado sobre el pronombre tú únicamente en los anuncios que se utilizan en las campañas de entes gubernamentales.
Por otro lado, en cuanto al nivel físico, no se presenta ninguna modificación del uso de pronombres de tratamiento, por lo que no se puede establecer ningún patrón que indique en qué momentos se utiliza alguno de los dos pronombres: su uso es completamente arbitrario.

Gráfica 4: uso de pronombres de tratamiento en el nivel discursivo

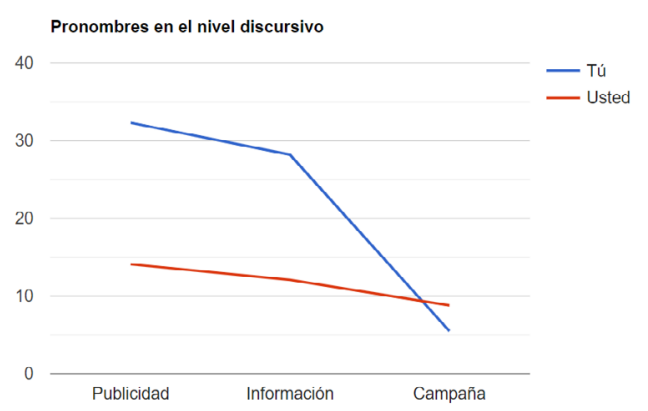

El uso de este pronombre (usted) en anuncios de campañas institucionales podría indicar que, al momento de presentar una información general sobre la ciudad o el estado de ésta, como calles cerradas, tipos de multas y zonas en construcción, el uso de usted se percibe como una mejor opción para comunicarse con los ciudadanos. Sin embargo, esta misma información presenta un problema, pues los datos obtenidos en el estudio del PLV de la gobernación del Quindío y la alcaldía de Armenia reflejan algo diferente. En ambos casos se usa en mayor medida el pronombre tú para comunicarse con los ciudadanos. Igualmente, esta contradicción se refleja en las entrevistas publicadas en las redes sociales de la alcaldía, en las cuales el alcalde, al momento de dirigirse a un interlocutor emplea el pronombre usted, pero cuando da 
información general y unidireccional emplea el pronombre tú, igual que en las redes sociales.

Es decir, se puede pensar que, en general, enelhablaarmeniaelpronombre tú se utiliza de manera unidireccional en anuncios publicitarios, invitaciones e información general que se da a los ciudadanos, pero de las cuales no se espera respuesta. Mientras que, cuando la comunicación es bidireccional, se procede a emplear el pronombre usted. Esta idea se presenta en la siguiente gráfica.

Gráfica 5: uso de pronombres de tratamiento con base en tipo de comunicación
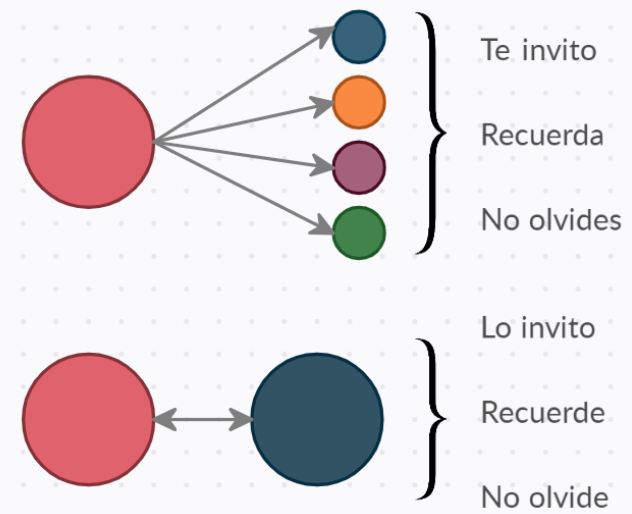

Finalmente, los datos obtenidos en esta investigación también presentan un hecho de curiosidad lingüística, pues como se evidenció en los apartados anteriores -imágenes 1, 6, 9 y 10 hay publicaciones en las cuales se hace uso de ambos pronombres de manera paralela para brindar la misma información, no hay variables que modifiquen su uso.

\section{Conclusión}

Esta investigación tuvo como objetivo describir la manera en la cual los pronombres de tratamiento se presentan en el paisaje lingüístico del departamento del Quindío (Colombia). Para la recolección y análisis de datos se diseñó una ruta metodológica que examinó los niveles físico y discursivo en el PL real, y las redes sociales de tres entidades regionales en el PL virtual. Siguiendo los resultados de estudios anteriores sobre el habla regional quindiana y el uso de pronombres de tratamiento en este departamento, como hipótesis se pensó que, si bien el pronombre característico del habla quindiana es usted, el uso de los pronombres en el PL debería coincidir. Los resultados obtenidos, sin embargo, refutan esta hipótesis.

En el PL se recolectaron 99 anuncios y carteles distribuidos en la ciudad de Armenia y se contaron 69 anuncios con el pronombre tú y 30 con el pronombre usted. Similarmente, en el análisis de las redes sociales de la gobernación del Quindío, la alcaldía de Armenia y el periódico regional $\mathrm{La}$ crónica del Quindío, se identificó que en los tres casos el pronombre de mayor uso es tú, siendo usado un $93.3 \%$ por la gobernación, un $86.6 \%$ por la alcaldía y un $63.3 \%$ por La crónica del Quindío. Además, se evidenciaron casos en los cuales, tanto en el PL como en el PLV, ambos pronombres se utilizan de manera paralela, ya sea en el mismo cartel o en la misma publicación.

Finalmente, tras comparar el uso de estos pronombres en el PL y en el PLV con los resultados obtenidos por Guarín (2020) y las entrevistas publicadas en las redes sociales mencionadas, se generó una nueva hipótesis sobre una nueva variable 
que puede modificar la alternancia de uso de los pronombres de tratamiento en esta región del país. Así pues, este artículo concluye que, por un lado, los pronombres usted y tú caracterizan el habla quindiana. Por otro lado, aunque en la oralidad el pronombre más empleado es usted, en el PL y en el PLV el pronombre de mayor uso es tú. Finalmente, esta investigación presenta la direccionalidad de la interacción como una nueva variable que modifica el uso de estos pronombres en el habla; en conversaciones bidireccionales la gente se comunica con el pronombre usted, a excepción de casos específicos como los mencionados en estudios anteriores, y utilizan tú para la comunicación unidireccional como las pancartas y los anuncios.

\section{Referencia bibliográfica}

Agudelo Montoya, C. L., Pasuy Guerrero, G. Y., Escobar Giraldo, O., \& Ramírez Osorio, J. F. (2016). Actitudes lingüísticas de los profesores de lenguas de la Universidad de Caldas respecto al voseo. Revista de Investigaciones UCM, 16(27), 16-28.

Amos, H. W., \& Soukup, B. (2020). Quantitative 2.0: Toward Variationist Linguistic Landscape Study (VaLLS) and a Standard Canon of LL Variables. En D. Malinowski \& S. Tufi (Eds.), Reterritorializing Linguistic Landscapes: $\quad$ Questioning Boundaries And Opening Spaces. Bloomsbury Academic.

Backhaus, P. (2007). Linguistic landscapes: A comparative study of urban multilingualism in Tokyo. Multilingual Matters.
Bartens, A. (2003). Notas sobre el uso de las formas de tratamiento en el español colombiano actual. 20. https://cvc.cervantes.es/lengua/ coloquio_paris/ponencias/pdf/ cve_bartens.pdf

Bengochea, N. (2019). Lenguas en la marquesina: Análisis del paisaje lingüístico de barrios de la Ciudad de Buenos Aires. Signo y seña, 35, 47-88. https://doi.org/10.34096/ sys.n35.6937

Ben-Rafael, E. (2009). A Sociological Approach to the Study of Linguistic Landscapes. En I. G. Shohami \& D. Gorter (Eds.), Linguistic landscape: Expanding the scenery (1st edition). Routledge.

Blommaert, J. (2013). Ethnography, Superdiversity and Linguistic Landscapes: Chronicles of Complexity. Multilingual Matters.

Brown, R., \& Gilman, A. (1970). The pronouns of power and solidarity. En T. Sebeok (Ed.), Style in Language (pp. 253-270).

Castillo Lluch, M., \& Sáez Rivera, D. M. (2013). Paisajes lingüísticos en el mundo hispánico. Revista Internacional de Lingüistica Iberoamericana (RILI), XI(1 (21)), 9-22.

Cepeda Ruiz, C. Y. (2014). Usted, tú, sumercé y vos: Formas pronominales de tratamiento en el español de Bogotá (Colombia) [Tesis de Maestría]. Universidad Nacional Autónoma de México.

Cisneros Estupiñán, M., Osorio Monsalve, S. M., \& Brito Cardona, S. (2014). El proyecto PRESEEA en la ciudad de Pereira, Colombia. XVII congreso internacional asociación de lingüística y filología de américa latina (ALFAL 2014), João Pessoa - Paraíba, Brasil. https://www. mundoalfal.org/CDAnaisXVII/ 
trabalhos/R0587-2.pdf

Colenso-Semple, S. (2008). ¿“Tú” o "usted"? La adaptación de los inmigrantes colombianos al sistema de tratamiento madrileño. Gaceta Hispánica de Madrid.

Comajoan Colomé, L., \& Long, E. (2012). The Linguistic Landscape of Three Streets in Barcelona: Patterns of Language Visibility in Public Space. En D. Gorter, H. F. Marten, \& L. Van Mensel (Eds.), Minority Languages in the Linguistic Landscape. Palgrave Macmillan UK. https://doi. org/10.1057/9780230360235

Coulmas, F. (2009). Linguistic Landscaping and the Seed of Public Sphere. En I. G. Shohami \& D. Gorter (Eds.), Linguistic landscape: Expanding the scenery (1st edition). Routledge.

Doyle D’Ambrosio, H. (2004). Pragmática, sociolingüística y pedagogía de los pronombres de tratamiento en lengua española. Estudios de Lingüística Aplicada, 39, 37-25.

Escobar, A. M., \& Potowski, K. (2015). El Español de los Estados Unidos. Cambridge University Press.

Extra, G., \& Barni, M. (2008). Mapping linguistic diversity in multicultural contexts: Cross-national and crosslinguistic perspectives. En M. Barni \& G. Extra (Eds.), Mapping linguistic diversity in multicultural contexts. Mouton de Gruyter.

Fernández Juncal, C. (2020). Funcionalidad y convivencia del español y el vasco en el paisaje lingüístico de Bilbao. Íkala, 25(3), 713-729. https://doi.org/10.17533/udea. ikala.v25n03a04

Flórez, L. (1953). Vos y la segunda persona verbal en Antioquian. Thesaurus : boletín del Instituto Caro y Cuervo, Tomo IX. Nums. 1, 2 y 3.
Franco Rodríguez, J. M. (2007). El español en el Condado de Miami-Dade desde su paisaje lingüístico. Linred, 5, 1-29.

García Rodríguez, A. (2014). Las fórmulas de tratamiento en el español de Colombia. https:// www.academia.edu/7394896/ L a s f\% C $3 \%$ B 3 rmulas de_tratamiento_en_el_ espa $\%$ C3\%B1ol_de_Colombia

Gorter, D. (2006). Introduction: The Study of the Linguistic Landscape as a New Approach to Multilingualism. En D. Gorter (Ed.), Linguistic landscape: A new approach to multilingualism. Multilingual Matters.

Gorter, D., \& Cenoz, J. (2017). Linguistic Landscape and Multilingualism. En J. Cenoz, D. Gorter, \& S. May (Eds.), Language Awareness and Multilingualism. Springer International Publishing. https:// doi.org/10.1007/978-3-31902240-6

Guarín, D. (2021). Uso de los pronombres de tratamiento en el Quindío. Artículo bajo revisión.

Guarín, D., Muriel Valencia, J., Pérez Gonzáles, J., \& Tabares Erazo, M. (2015). Descripción lingüística del apodo en el habla cotidiana de futuros docentes bilingües licenciados en Lenguas Modernas. En A. Galindo Martínez (Ed.), Investigación en Bilingüismo: Aportaciones de futuros licenciados en Lenguas Modernas de la Universidad del Quindio (Vol. 1, pp. 109-127). Editorial Kinesis. 
Hipperdinger, Y. H. (2020). Lenguas e imágenes en la cartelería comercial: Concurrencias en un paisaje lingüístico argentino. Revista de Filología de la Universidad de La Laguna, 41, 161-182. https://doi. org/10.25145/j.refiull.2020.41.08

Idárraga Valencia, S. J. (2017). La investigación sociolingüística en la Universidad del Quindío. Boletín Redipe, 6(10), 32-41.

Ivkovic, D., \& Lotherington, H. (2009). Multilingualism in Cyberspace: Conceptualizing The Virtual LinguisticLandscape.International Journal of Multilingualism, 6(1), 17-36. https://doi. org/10.1080/14790710802582436

Jang, J. S. (2012). La dinámica de la alternancia entre tú, vos y usted en Medellín (Colombia) desde la teoría de la acomodación comunicativa. Forma y Función, 25(1), 22.

Jang, J. S. (2014). La frecuencia del uso del usted en tres zonas del departamento de Antioquia (Colombia). Forma y Función, 27(1), 45-65. https://doi. org/10.15446/fyf.v27n1.46943

Kasanga, L.A. (2015). Semiotic Landscape, Code Choice and Exclusion. En R. Rubdy \& S. Ben Said (Eds.), Conflict, Exclusion and Dissent in the Linguistic Landscape. Palgrave Macmillan.

Lara Bermejo, V. (2018). Acomodación en los pronombres de tratamiento de ecuatorianos en España. Lengua y migración, 10(1), 7-31.

Leimgruber, J., Vingron, N., \& Titone, D. (2020). What Do People Notice from Real-World Linguistic Landscapes? A Review of the Literature and Recommendations for Future Eye-Tracking Research. En D. Malinowski \& S. Tufi (Eds.), Reterritorializing Linguistic
Landscapes:

Questioning Boundaries and Opening Spaces. Bloomsbury Academic.

Lindgård, A. (2016). El ustedeo de familiaridad en Colombia [Tesis de Maestría]. Universidad de Tromsø.

López López, G. (2015). Diferencias dialectales en el uso de las formas de tratamiento: Tú y usted en páginas de Facebook de dos equipos de fútbol de Bogotá. 4to Encuentro internacional de español como lengua extranjera, Bogotá, Colombia. https://spanishincolombia. c a ro y c u e r vo.gov.co/ encuentro-lengua-extranjera/ ponencias/L $\% \mathrm{C} 3 \% \mathrm{~B} 3$ pez $\% 20$ L\%C3\%B3pez\%20Giovani.pdf

Ma, Y. (2019). El paisaje lingüístico chinoespañol en la ciudad de Valencia y su aplicación a la enseñanza de ELE a los sinohablantes [Tesis Doctoral]. Universidad de Valencia.

Maldonado Sandoval, Á. M. (2020). El Paisaje Lingüístico de la ciudad de Bogotá como recurso pedagógico en la clase de ELE [Tesis de Maestría, Universidad Nacional de Colombia]. https://repositorio. unal.edu.co/handle/unal/77836

Mas Álvarez, I. (2014). Formas de tratamiento y enseñanza del español como lengua extranjera. redELE: revista electrónica de didáctica del español lengua extranjera, 26.

Montes Giraldo, J. J. (1982). El español de Colombia: Propuesta de clasificación dialectal. Thesaurus: boletin del Instituto Caro y Cuervo, 37(1), 23-92.

Montes Giraldo, J. J. (1985). Estudios sobre el español de Colombia. Instituto Caro y Cuervo. 
Mooney, A., \& Evans, B. (2015). Language, Society and Power (4a ed.). Routledge. https://doi. org/10.4324/9781315733524

Ospina Castro, L. (2018). Las formas de tratamiento en el español colombiano y su reflejo en algunos manuales de E/LE [Trabajo Final de Master]. Universitat de Barcelona.

Rivera-Mills, S. (2011). Use of Voseo and Latino Identity: An Intergenerational Study of Hondurans and Salvadorans in the Western Region of the U.S. En Selected Proceedings of the 13th Hispanic Linguistics Symposium. Cascadilla Proceedings Project.

Rubdy, R. (2015). Conflict and Exclusion: The Linguistic Landscape as an Arena of Contestation. En R. Rubdy \& S. Ben Said (Eds.), Conflict, Exclusion and Dissent in the Linguistic Landscape. Palgrave Macmillan.

Sebba, M. (2011). Discourses in Transit. En A. Jaworski \& C. Thurlow (Eds.), Semiotic landscapes: Language, image, space. Continuum.

Serna Pinto, M. A. (2018). La modalidad en los morfemas de futuro en el corpus PRESEEA- Pereira. https://www. academia.edu/36575881/The modality_in_the_morphemes_of future_in_the_corpus_PRESEEA Pereira

Spolsky, B. (2009). Prolegomena to a Sociolinguistics Theory of Public Signage. En I. G. Shohami \& D. Gorter (Eds.), Linguistic landscape: Expanding the scenery (1st edition). Routledge.
Thistlethwaite, J., \& Sebba, M. (2015). The Passive Exclusion of Irish in the Linguistic Landscape: A Nexus Analysis. En R. Rubdy \& S. Ben Said (Eds.), Conflict, Exclusion and Dissent in the Linguistic Landscape. Palgrave Macmillan.

Uber, Diane R. (2011). Forms of Address: The Effect of the Context. En M. Díaz-Campos (Ed.), The Handbook of Hispanic Sociolinguistics (pp. 244-262). Wiley-Blackwell. https://doi. org/10.1002/9781444393446.ch12

Uber, Diane Ringer. (1985). The Dual Function of usted: Forms of Address in Bogotá, Colombia. Hispania, 68(2), 388-392.

Weyers, J. R. (2016). Medellín Cuenta con vos: The changing role of voseo in written communication. Comunicación, 37, 67-81. 